Thorax (1966), 21, 529.

\title{
Fixation of aortic ball valve prosthesis (Starr-Edwards)
}

\author{
VIKING OLOV BJÖRK AND GÖSTA HULTQUIST \\ From the Departments of Thoracic Surgery and Pathology, University Hospital, Uppsala, Sweden
}

The technique of fixation of a ball valve prosthesis in the area of the non-coronary cusp by bringing the sutures from the collar of the prosthesis out through the wall of the aorta and tying them over strips of dacron has been discussed by Starr, Edwards, McCord, and Griswold (1963), and advocated by Effler, Groves, and Favaloro (1964). We have never in our first 100 operated cases seen a leak in the area of the non-coronary cusp when using internal fixation through the base of the noncoronary cusp. On the other hand, a weak area seems to exist in the right and left coronary cusps close to the commissure between these two cusps, where we have encountered leakage at the side of the prosthesis in three non-infected and nine infected cases. The aim of this paper is to report the different anatomy found at the base of the different cusps in the root of the aorta with special reference to the fixation of the ball valve prosthesis.

\section{METHOD}

A series of vertical sections has been made through the aortic ostium (Fig. 1) through the centre of each cusp (A, C, E) as well as through the commissure between the cusps (B, D, F). From these sections a drawing of the structure has been made, slightly enlarged, in a projection apparatus (Fig. 2, A-F).

\section{RESULTS}

From this investigation it is evident that the connexion between the base of the aortic cusps and the septal myocardium of the left ventricle has a different structure in different parts of the circumference of the aorta. In the base of the non-coronary cusp (Figs 1 and 2, D, E, F) there is more tight, tendinous tissue between the origin of the cusp and the myocardium. Corresponding to the base of the left and right aortic cusps and the commissure between them (Figs 1 and 2, A-C) the length of connective tissue

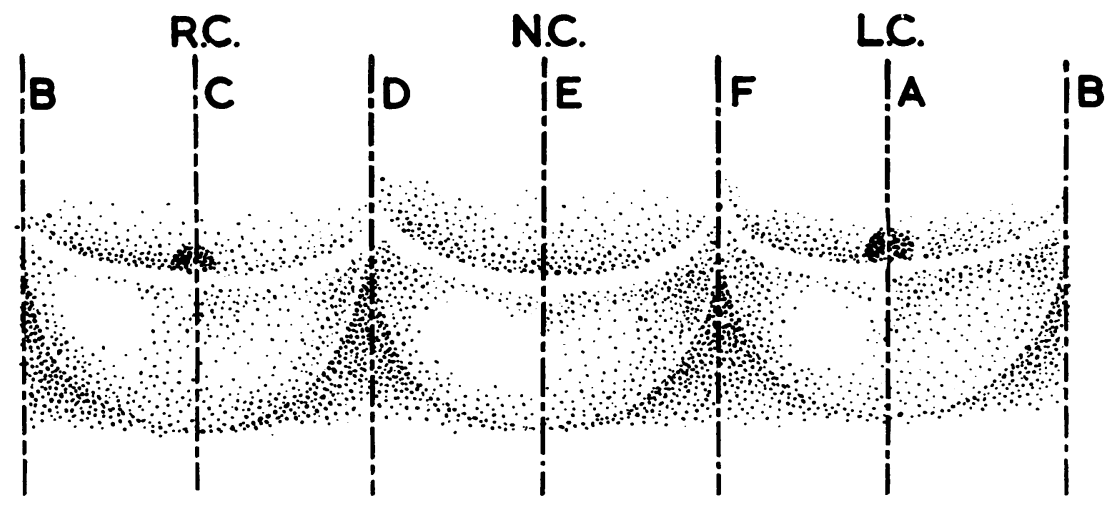

FIG. 1. Diagram of the position of the vertical sections through the root of the aorta shown in Fig. 2 ; (A) through the centre of the left coronary cusp (L.C.); $(B)$ through the commissure between the left and right coronary cusps; $(C)$ through the centre of the right coronary cusp (R.C.); (D) through the commissure between the right and the non-coronary aortic cusps; $(E)$ through the centre of the noncoronary aortic cusp (N.C.); (F) through the commissure between the non-coronary and the left coronary aortic cusps. 


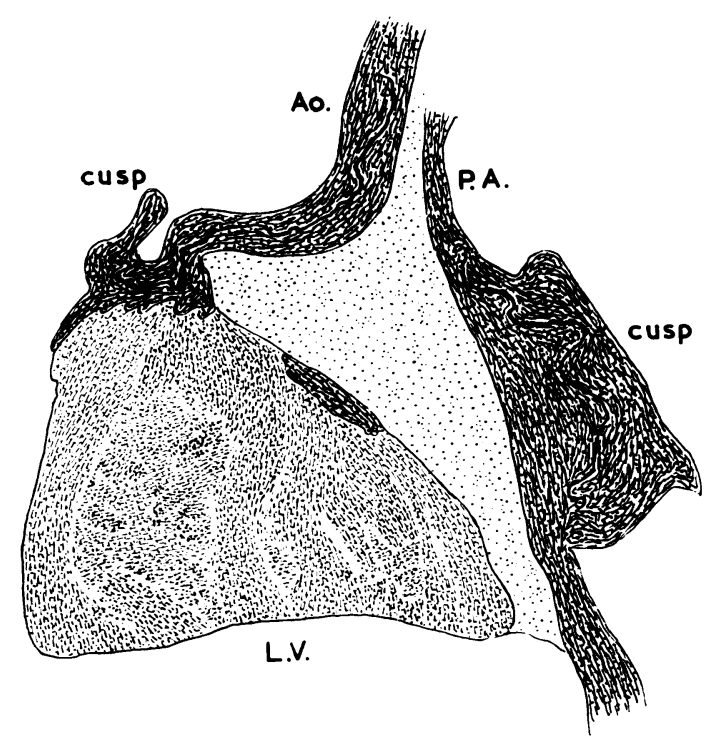

FIG. 2A

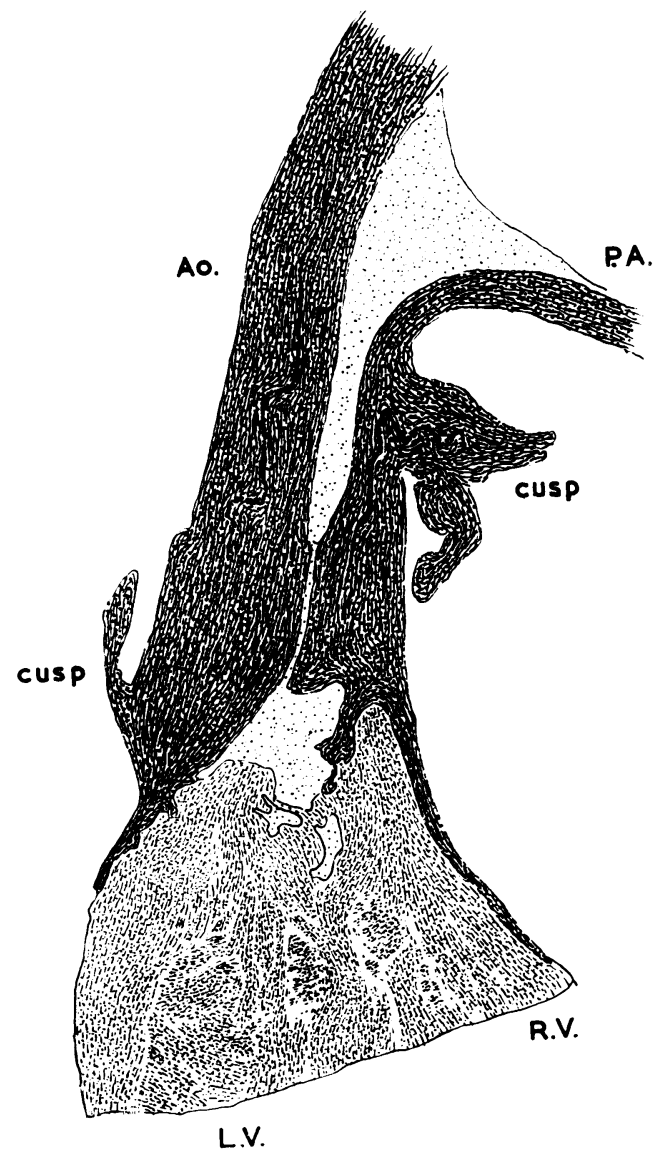

FIG. 2B

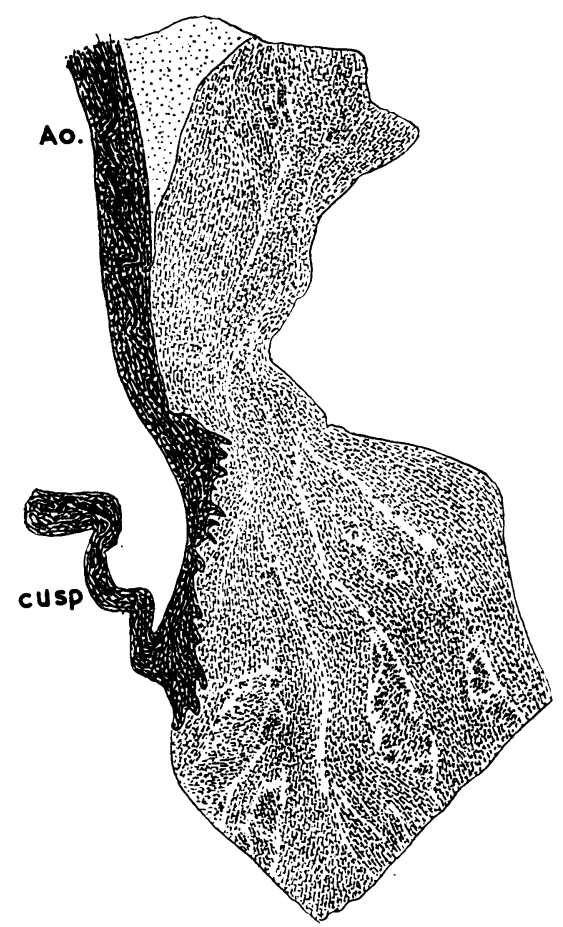

FIG. 2C

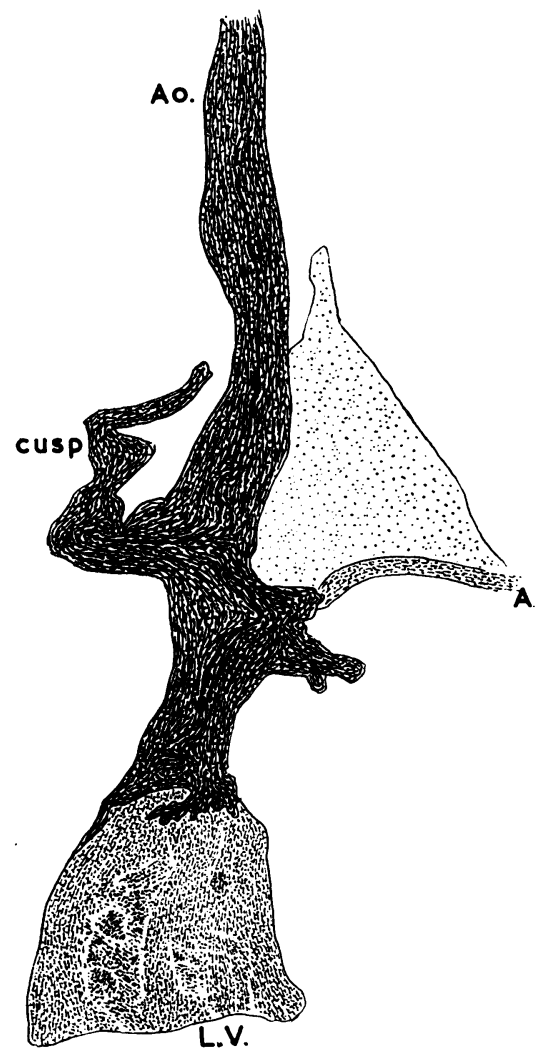

FIG. 2D 


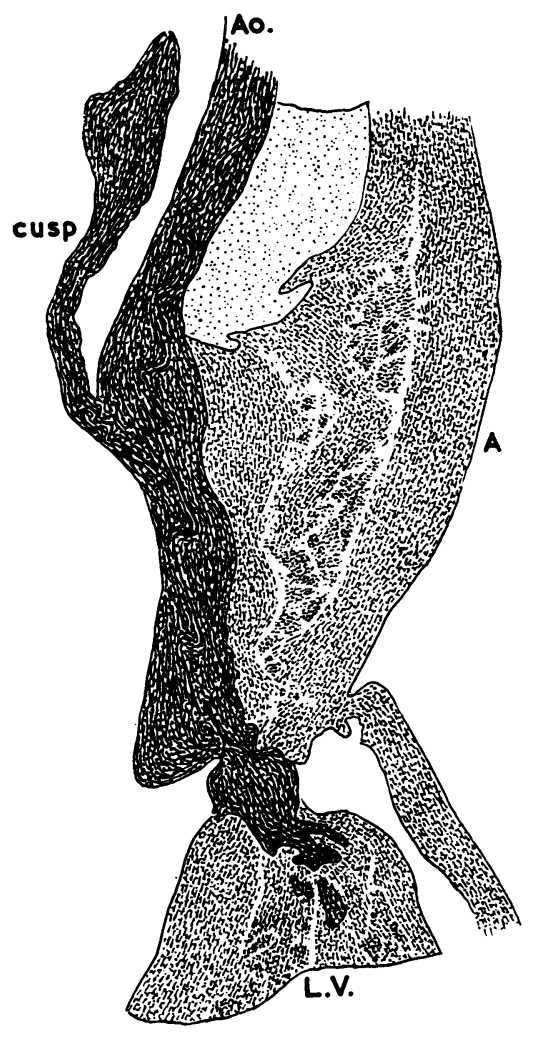

FIG. 2E

FIG. 2. (A) Centre of the left coronary aortic cusp. (B) Commissure between the left and right coronary cusps. (C) Centre of the right coronary cusp. (D) Commissure between the right and non-coronary aortic cusps. (E) Centre of the non-coronary aortic cusp. (F) Commissure between the non-coronary and left aortic cusps. Ao.=aorta; P.A.=pulmonary artery ; R.V.=right ventricle; L.V.=left ventricle; $A=$ atrium.

between the origin of the cusp and the myocardium is shorter, and the connective tissue is joined to the septal myocardium by a broad plate.

\section{DISCUSSION}

Cineangiographic investigation has shown that the movement of the base of the ball valve prosthesis is less pronounced in the area of the non-coronary cusp. Furthermore, this histological investigation

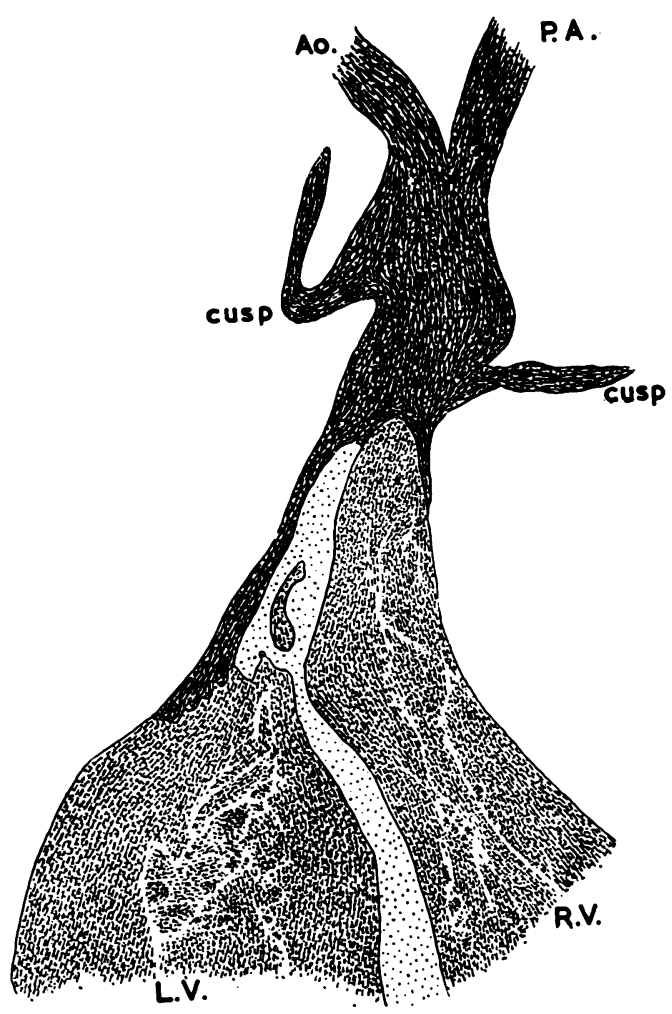

FIG. 2F

Fat and loose connective tissue

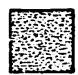

Myocardium

Tendinous connective tissue and the wall of the aorta and the pulmonary artery

has shown a difference in the structure of the root of the aorta with a much longer length of tough connective tissue between the origin of the non-coronary cusp and the myocardium. Which of these two factors has been more important in the prevention of suture insufficiency corresponding to the base of the non-coronary cusp is difficult to evaluate. The favourable structural arrangement of connective tissue between the base of the non-coronary cusps and the myo- 


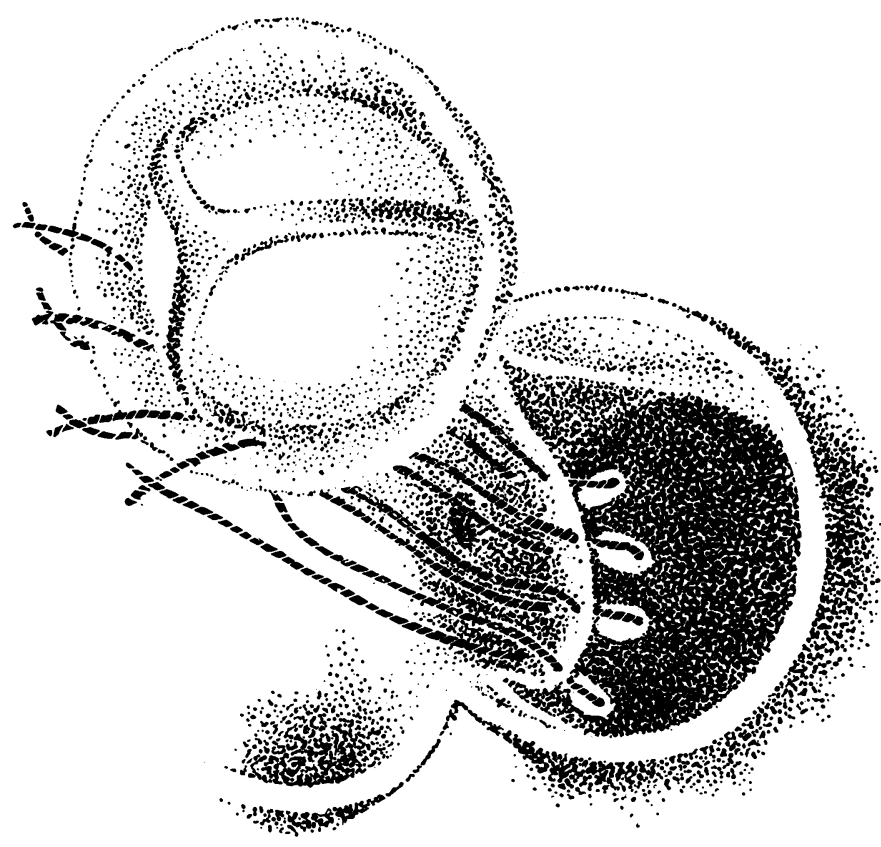

FIG. 3. Diagram of the fixation tech- $\frac{\overline{\bar{\sigma}}}{\frac{\sigma}{\sigma}}$ nique with mattress sutures buffered with $\stackrel{\mathbb{Q}}{\varrho}$ teflon pearls through the remnant of the in base of the aortic cusp. cardium is ideal for internal fixation of the collar of the ball valve prosthesis. Experience has confirmed this view, and out of a total 12 cases of suture insufficiency none was found in the area of the non-coronary cusp. In one with bloodstream infection, two-thirds of the circumference of the ball valve prosthesis had become loose; but all the sutures in the area of the non-coronary cusp were still intact. In view of our experience and the findings demonstrated, we do not advocate fixation of the ball valve prosthesis by sutures through the wall of the aorta but favour instead internal fixation with mattress sutures buffered with teflon pearls on the ventricular side and through the remnant of the base of the noncoronary aortic cusp to the collar of the prosthesis. The same technique is also used for the right and left coronary cusps, care being taken to avoid deep bites and to place the silk sutures at 2 to $3 \mathrm{~mm}$. distance from each other (Fig. 3).
SUMMARY

Histological investigation has revealed a longer $\stackrel{2}{\stackrel{2}{\varrho}}$ length of tendinous tough connective tissue $\overrightarrow{\vec{P}}$ between the origin of the non-coronary cusp and $\frac{0}{3}$ the myocardium as compared with the right and left coronary cusps. We therefore favour internal: fixation of the ball valve prosthesis, in the area of the non-coronary cusp, with mattress sutures 음 buffered with teflon pearls on the ventricular side $\underset{x}{\varnothing}$ through the base of the non-coronary aortic cusp. $\frac{0}{3}$ Our experience has confirmed the opinion that external fixation of a ball valve prosthesis through 8 the root of the aorta corresponding to the noncoronary cusp is of no advantage.

\section{REFERENCES}

Effler, D. B., Groves, L. K., and Favaloro, R. (1964). Aortic and mitral valve replacement: experience with Starr-Edwards prostheses. Arch. Surg., 88, 145.

Starr, A., Edwards, M. L., McCord, C. W., and Griswold, H. E. (1963). Aortic replacement. Clinical experience with a semirigid $N$ ball-valve prosthesis. Circulation, 27, 779. 